\title{
PAESAGGI ED ECONOMIE TRA ROMA E IL MARE. LA SFIDA DELLA SOSTENIBILITÀ 1
}

\author{
KETI LELO \\ keti.lelo@,uniroma3.it \\ UNIVERSITÀ ROMA TRE, CROMA \\ ANNA LAURA PALAZZO \\ annalaura.palazzo@uniroma3.it \\ Università Roma Tre, Dipartimento di ARCHITETTURA \\ http://dx.doi.org/10.25267/Riparia_sup.2018.i1.10
}

\section{Uno sguardo d'insieme}

A partire dagli anni Settanta, il territorio del Lazio è stato interessato da significativi fenomeni di redistribuzione - diffusione, trasferimento e riorganizzazione - delle attività di produzione e servizio con un imponente consumo di suolo. Cifra di questo paradigma è la localizzazione ubiquitaria della piccola impresa, per sua natura più flessibile, che ha reso via via obsoleti gli spazi riservati alla produzione fordista: le aree attrezzate della ex-Cassa del Mezzogiorno (ASI), e i Piani per gli insediamenti produttivi, che peraltro nel Lazio avevano avuto scarso rilievo sin dall'origine.

Le interpretazioni di questi processi e dei funzionamenti che li sottendono non sono univoche: si sostiene ad esempio il progressivo ingresso, sulla scena di un territorio che si è fatto 'Città-regione', dei comuni di prima e seconda cintura depositari di attività e funzioni di rango che hanno progressivamente occupato nell'ultimo trentennio il tenace deserto intorno alla Capitale con consistenti espansioni ${ }^{2}$. Una seconda interpretazione, suffragata dalle testimonianze degli operatori economici, sottolinea nuove connessioni tra aree sub-regionali in grado di scambiare reciprocamente merci e persone con un affaccio su mercati esteri in crescente autonomia dal richiamo di Roma. Una terza interpretazione mostra la persistenza di un modello centripeto legato al forte richiamo della Capitale: 1' $83 \%$ di tutto il capitale estero investito tra 2003 e 2014, e il 93\% nella creazione di nuovi posti di lavoro nel Lazio hanno come destinazione Roma.

Se il destino economico dell'area romana è sempre più evidentemente modellato da fattori esterni ed interessi esogeni, il passaggio dell'organismo urbano da sistema chiuso a sistema aperto, passaggio tematizzato e programmato in altre città europee, è stato solamente evocato nell'ultima stagione di pianificazione. Di fatto, l'avvio della città metropolitana, ai sensi della Legge n.56 del 7 aprile 2014, Disposiżoni sulle città metropolitane, sulle province, sulle unioni e fusioni di comuni, porrà all'agenda della governance dei 112 comuni della soppressa Provincia su un'area di $5.352 \mathrm{kmq}$ pressanti questioni relative a traguardi di sostenibilità e resilienza: da un lato, una

\footnotetext{
${ }^{1} \mathrm{Il}$ progetto e i contenuti del presente contributo sono di comune responsabilità. Il paragrafo 1 è da attribuire ad Anna Laura Palazzo e il paragrafo 2 a Keti Lelo.

${ }^{2}$ Con riferimento al territorio della ex-Provincia, gli abitanti sono 4.330.000. Entro sessanta chilometri dalla capitale sorgono nel 2011 (fonte: Censimento) sette centri con una popolazione compresa tra i 50.000 e i 70.000 abitanti: Guidonia, Fiumicino, Aprilia, Pomezia, Tivoli, Velletri, Anzio; altri quattordici hanno una popolazione compresa tra i 20.000 e i 50.000 abitanti: Nettuno, Ardea, Monterotondo, Marino, Albano Laziale, Ladispoli, Ciampino, Cerveteri, Fonte Nuova, Genzano di Roma, Colleferro, Mentana, Frascati e Palestrina.
} 
redistribuzione delle funzioni di rango e del sistema dell'accessibilità, dall'altro, uno statuto inclusivo di 'territorio aperto' che si estenda alle 'infrastrutture pubbliche di natura', sia entro gli strumenti di pianificazione delle aree protette, che nella dimensione culturale e ricreativa delle nuove pratiche di loisir e agricoltura periurbana.

Su questo sfondo, il territorio compreso nella piana del Tevere tra Roma e il mare risulta tra i più dinamici, ed è composto di varie realtà dai caratteri identitari ben distinti. Sede di grandi attrattori per la mobilità e le funzioni di rango (l'Aeroporto e il Porto di Fiumicino, la Nuova Fiera di Roma, diversi centri direzionali), e come tale aperto alle forze della globalizzazione e del mercato, mantiene qualche margine di autonomia dalla prorompente centralità della Capitale.

L'originaria condizione di qualità percepita associata alla vicinanza del mare e di spazi naturali di pregio assoluto - la pineta, le aree archeologiche, insediamenti organizzati a bassa densità -, è stata motivo di una crescita residenziale estremamente sostenuta. Tale qualità è stata progressivamente scalfita dall'incremento dei carichi urbanistici, che escludono oggi, pur nella dimensione definitivamente urbana del 'vivere diffuso', l'aggancio a prestazioni locali soddisfacenti in termini di attrezzature e servizi alla persona, obbligando a comportamenti molecolari scanditi dal pendolarismo e dall'uso dell'automobile.

Diffusione e dispersione insediativa mettono a dura prova la sopravvivenza di un sistema ambientale fragile (falda superficiale, esondazioni del Tevere) e dall'inestimabile valore storico e naturalistico, nonostante o forse proprio a causa di regimi di pianificazione articolati e complessi (Piano Paesaggistico, Piano Territoriale Provinciale, Piano di Bacino, Piano di gestione della Riserva del Litorale romano, Parco archeologico del Porto di Traiano-Necropoli di Porto-Ostia Antica).

Qui, la sfida della sostenibilità incorpora innanzitutto la dimensione ambientale, attraverso la salvaguardia di lembi residuali di naturalità insospettata, la tutela delle trame agricole della bonifica novecentesca, la valorizzazione delle risorse storiche, interpellando oltre agli attori istituzionali le pratiche di modificazione minuta dello spazio dell'abitare, le progettualità che si annidano nell'agire dei soggetti e il connotato spaziale di questo agire, che si traduce nella qualità del patto sociale (equità). La governance dello sviluppo territoriale tra Roma e il mare - un 'progetto di territorio', - dovrebbe puntare anche alla qualità delle prestazioni erogate (efficienza), orientando verso il miglioramento dell'offerta di attività e servizi avanzati, rafforzando le relazioni di mutua utilità con il sistema insediativo, le infrastrutture, l'ambiente e il paesaggio.

\section{Motivazioni, razionalità e trend insediativi}

Un forte impulso alla trasformazione del quadrante tra Roma e il mare si verificò a partire dall'Unità d'Italia, quando la navigabilità del tratto terminale del Tevere apparve congeniale all'avvenire di una città che si apriva cautamente a uno sviluppo industriale lineare. L'avvio di imponenti opere di infrastrutturazione risale all'operato del primo ventennio del secolo XX con il ripristino della Via Ostiense, la costruzione della ferrovia Roma-Lido e significativi studi per un canale navigabile, e trova una ideale continuazione sotto il ventennio fascista, con la realizzazione della Via del Mare (1927-28), la costruzione della stazione ferroviaria Ostiense (1931), la progettazione dell'E42 come avamposto urbano destinato ad ospitare l'Esposizione universale del 1942. Nel secondo dopoguerra, proseguono i lavori con la metropolitana di collegamento tra Termini e l'EUR (inaugurata nel 1955), l'aeroporto di Fiumicino (1960) e l'Autostrada di collegamento che, col passare degli anni, ha 
acquisito un ruolo sempre maggiore di supporto alle attività economiche dell'intero quadrante.

Frattanto, l'espansione residenziale si appoggerà soprattutto alla via Cristoforo Colombo, realizzata negli anni '40 per collegare l'EUR al centro di Roma e al mare (fig. 1).

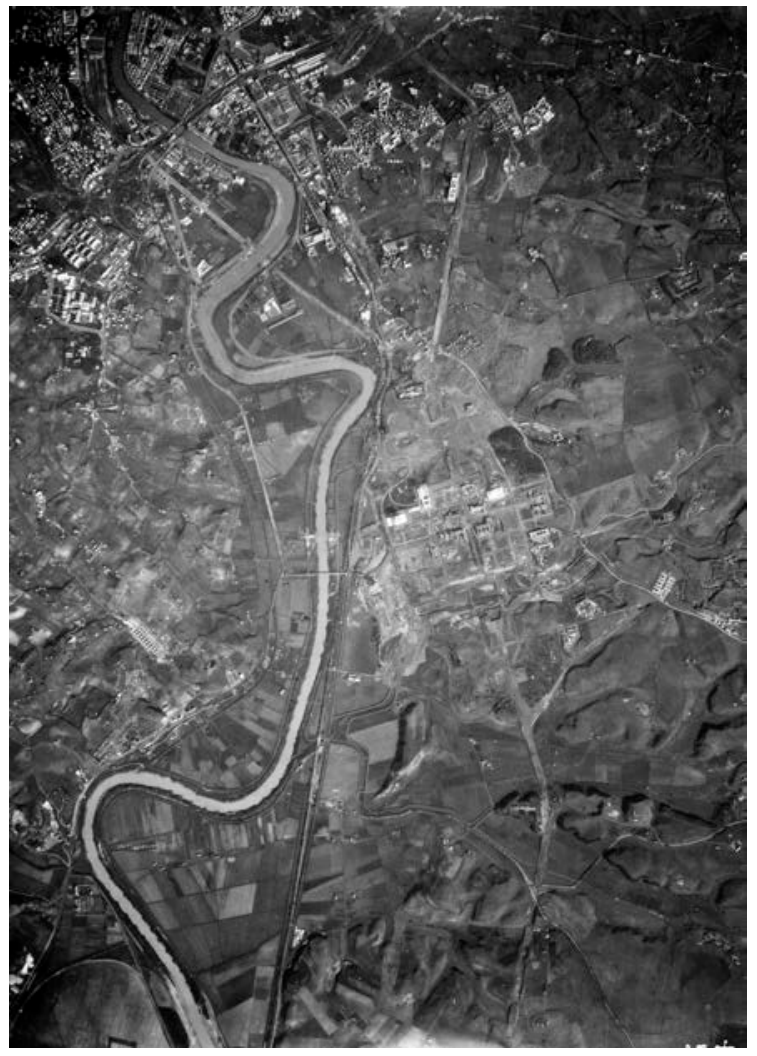

Fig 1. Il tracciato di via Cristoforo Colombo dalla città verso il mare, 1941. L'ultima propaggine della città consolidata verso sud è costituita dalla città giardino Garbatella (a destra della Colombo). Si noti come la Colombo connetta la città con il quartiere in costruzione dell'Eur.

Il quadrante urbano ospita oggi un vero e proprio mosaico di tipologie insediative. Accanto ai quartieri 'storici' come la Garbatella o l'EUR, ormai parte della città consolidata, vi sono esempi rilevanti di intervento pubblico come il quartiere INCIS di Decima, costruito negli anni Sessanta su progetto di Luigi Moretti, oppure i quartieri PEEP di Corviale e Laurentino 38, realizzati a cavallo tra gli anni Settanta e Ottanta, nella cui impostazione erano presenti elementi sperimentali e anche utopistici che ne hanno profondamente segnato il destino.

Ai massicci interventi 'ufficiali', come Spinaceto (PEEP), Acilia, Dragoncello, Portuense (previste nella Variante integrativa al Piano regolatore del 1981), si contrappongono interventi di edilizia privata, come il quartiere Magliana Nuova, costruito - in accordo con il Comune - in un'ansa del Tevere, o il cosiddetto 'quartiere satellite' di Casalpalocco, ambizioso progetto realizzato tra l'inizio degli anni Sessanta e la metà dei Settanta dalla Società generale immobiliare, che proponeva ai ceti medi e superiori un modello di residenza ispirato all'american way of life. 
Nelle ultime tre decadi, l'intervento pubblico è inesistente, mentre dilagano i nuovi quartieri di iniziativa privata edificati oltre i confini del Grande Raccordo Anulare, configurati come mix funzionali. Ne è un esempio l'insediamento di Parco Leonardo nei pressi dell'aeroporto di Fiumicino, che coniuga le funzioni residenziali con quelle commerciali e ricreative.

La dispersione insediativa ha profondamente modificato i caratteri del sistema morfologico-ambientale. Isola Sacra, Ostia Antica, Casal Palocco, Infernetto, Dragoncello, Acilia, Centro Giano, Casal Bernocchi, Vitinia, sono ormai un continuum di insediamenti che si addensano lungo le principali direttrici di collegamento con Roma, per poi disperdersi nel territorio, seguendo i percorsi perpendicolari disegnati dalla maglia della bonifica.

I consumi del suolo sono in crescita costante, ma l'utilizzazione è limitata: infatti, in più di un terzo delle aree residenziali, il coefficiente di occupazione del suolo (rapporto tra superficie coperta e superficie perimetrata) risulta inferiore al $10 \%$. Esempi significativi, proprio in virtù della loro estensione, riguardano le recenti urbanizzazioni di Piana del Sole e Valleranello.

L'eccezionale dotazione infrastrutturale dell'area e la sua posizione strategica l'hanno resa negli ultimi decenni particolarmente appetibile per le funzioni terziarie e direzionali. In particolare, il settore lungo la direttrice Roma-Fiumicino, è stato investito dalla presenza di grandi complessi orientati prevalentemente alla direzionalità, al tempo libero, e al commercio all'ingrosso. Senza volere essere esaustivi, solo nella fascia compresa tra Via Portuense e Via Ostiense si possono citare: il Centro Direzionale Alitalia-Centro Toyota (2002)-Hotel Ramada (2004); The Village-Parco de' Medici (2001); Commercity (2003); Cineland (1999); la Nuova Fiera di Roma (2006).

In concomitanza con questo viraggio nella caratterizzazione del quadrante urbano, ha progressivamente preso piede il cosiddetto 'settore creativo', che la letteratura internazionale inquadra tra $\mathrm{i}$ principali fattori di competitività nell'economia della conoscenza, suscettibile di produrre reddito e riprodurre forza lavoro: le cui preferenze localizzative sono spia di elevata qualità della vita, legata in questa circostanza alla disponibilità di spazi naturali di grande valore e a buoni standard di accessibilità.

La definizione assunta per la presente mappatura dell'area metropolitana di Roma e del quadrante prescelto (fig. 2) corrisponde con quella proposta dal National Endowment for Science, Technology and the Arts (NESTA), che assume come industrie creative '...quelle attività che hanno origine nella creatività e talento individuale, e che hanno il potenziale di creare lavoro e ricchezza attraverso la generazione e lo sfruttamento delle proprietà intellettuali'. Le attività sono classificate in 'strati', che possono essere interpretati come elementi di una catena del valore creativo: quelle legate alla creazione di contenuti si trovano al 'cuore' della catena, mentre quelle legate alla produzione, distribuzione e commercio, sono collocate in 'periferia's.

${ }^{3}$ I dati utilizzati per analizzare le imprese creative provengono dall'Archivio Statistico delle Unità Locali delle Imprese Attive (ASIA-UL), forniti dall'Istituto Nazionale di Statistica (ISTAT). Si tratta di un registro delle imprese aggiornato annualmente attraverso un processo di integrazione tra fonti amministrative e fonti statistiche. Esso fornisce informazioni di identificazione (nome e indirizzo), sull'attività economica (codice ATECO) e sulla struttura 


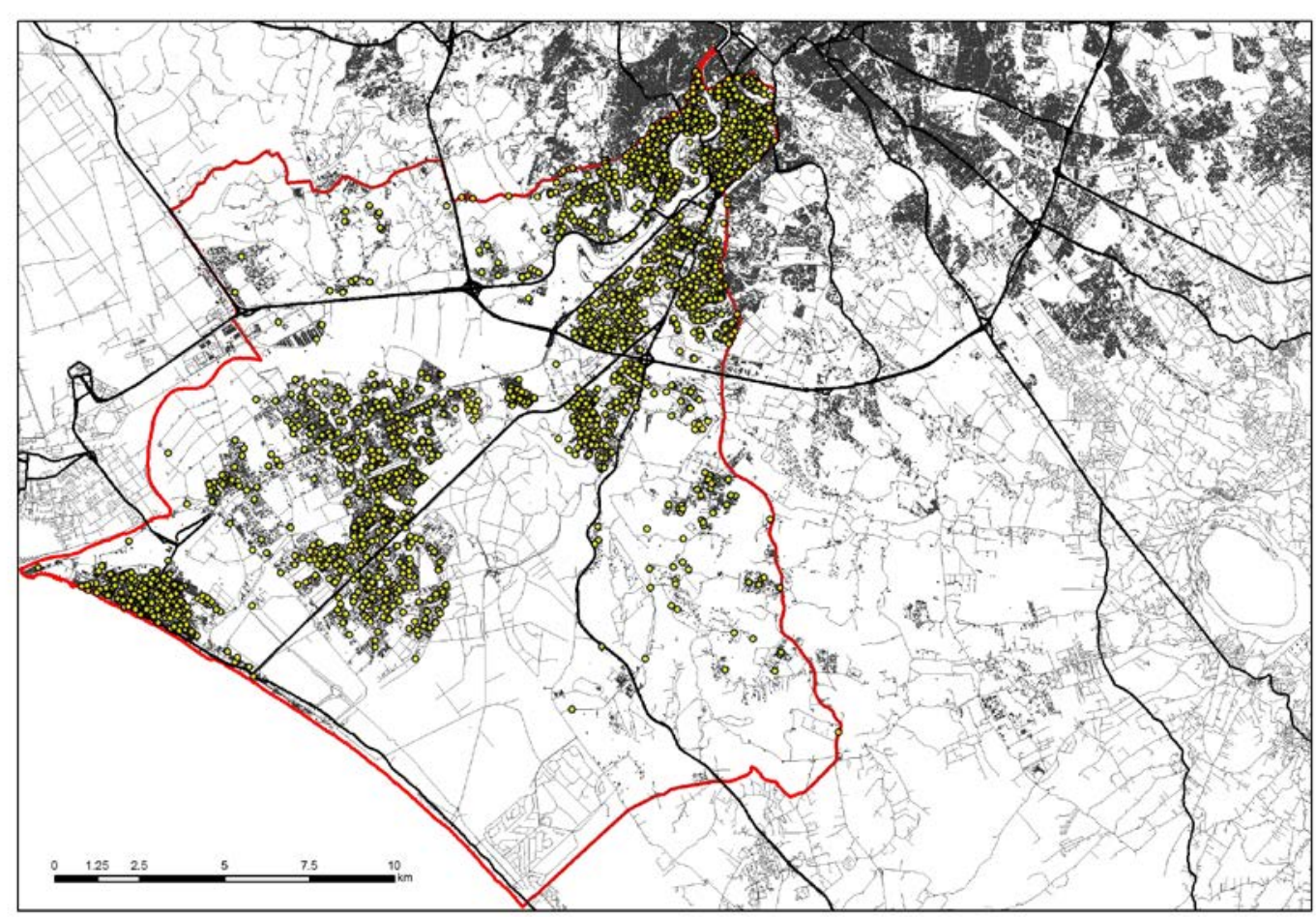

Fig. 2. Localizzarione delle imprese creative nell'area metropolitana di Roma, 2009. Fonte: ISTAT ASIA-UL.

Con riferimento ai dati di Tabella 1, la caratteristica più marcata è la forte presenza di imprese che operano nel settore della produzione di software e videogiochi. Esse rappresentano, infatti, circa il $50 \%$ del totale numero di imprese creative e il $70 \%$ degli addetti nell'area metropolitana.

Tabella 1. Peso di ogni categoria creativa nel territorio tra Roma e il mare rispetto all'area metropolitana (2009).

\begin{tabular}{lll}
\hline Categorie creative & $\%$ imprese & $\%$ addetti \\
\hline Architettura & 11.41 & 17.79 \\
Antichità e artigianato & 8.10 & 9.60 \\
Comunicazione e branding & 11.71 & 13.13 \\
Design & 10.18 & 12.12 \\
Editoria & 6.13 & 10.58 \\
Musica e arti performative & 11.87 & 11.74 \\
Radio e TV & 7.19 & 0.79 \\
Videogiochi e software & 46.10 & 70.26 \\
Video, film e fotografia & 3.58 & 1.67 \\
\hline Totale & 11.58 & 15.85 \\
\hline Fonte: ISTAT ASIA-UL 2009.
\end{tabular}

Fonte: ISTAT ASIA-UL 2009.

aziendale (lavoratori dipendenti e indipendenti, forma giuridica, fatturato) delle unità locali delle imprese. 
In maniera del tutto analoga con ciò che si osserva a livello metropolitano, l'80,33\% delle attività economiche nei settori creativi è rappresentato da imprese con un singolo addetto; il 17,21\% delle imprese ha tra 2 e 20 addetti; il 1,31\% delle imprese ha tra 21 e 50 addetto, e 1,15\% delle imprese ha più di 50 addetti (fig. 3). La distribuzione delle imprese creative per classe di addetti nell'area metropolitana (A) e tra Roma e il mare (B), evidenzia che, sebbene le quote di imprese nelle varie categorie dimensionali rimangano simili (cerchio esterno dei grafici A e B), c'è una maggiore presenza di addetti nella fascia dimensionale più elevata (cerchio interno dei grafici A e B), che depone per una presenza tra Roma e il mare di imprese con numero di addetti maggiore rispetto alla media metropolitana.

A

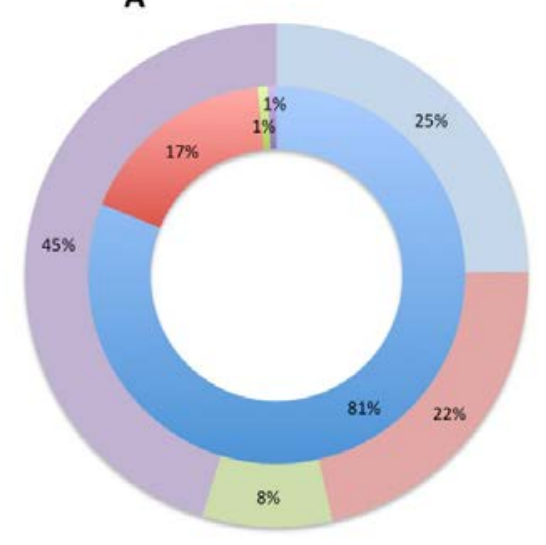

B

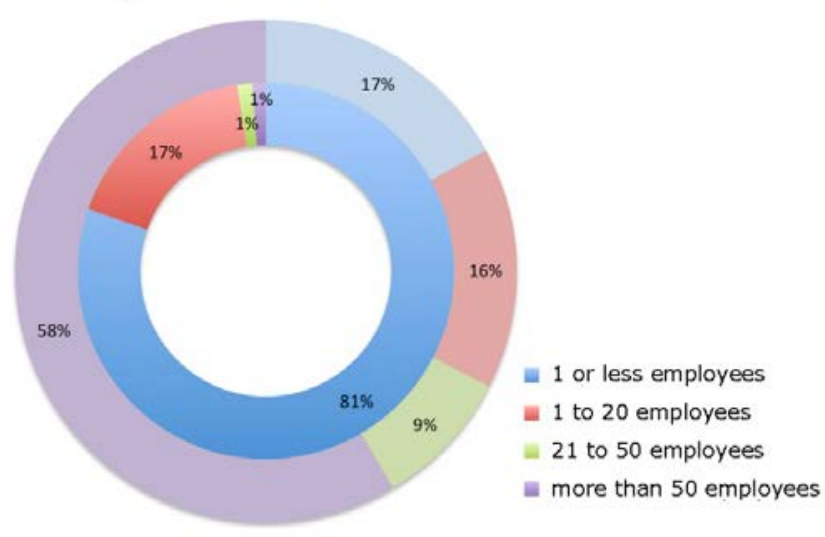

Fig. 3. Distribuzione delle imprese creative per classe di addetti (2009).

A) nell'area metropolitana; B) tra Roma e il mare. Il cerchio interno rappresenta il numero di imprese creative mentre quello esterno in numero di addetti.

L'evidenza nell'area di un 'cluster' così economicamente rilevante di imprese creative, che allo stato delle conoscenze non consentono di decifrare eventuali modalità di integrazione orizzontali o verticali o anche semplici razionalità di funzionamento al proprio interno, può costituire uno stimolante punto di partenza per ulteriori indagini volte all'individuazione di quelle caratteristiche e vocazioni del territorio che, opportunamente inserite nelle politiche territoriali, possono contribuire al miglioramento delle sue performance socio-economiche.

\section{Bibliografia}

R. CASSETTI, Roma e il Lazio 1945-2007. La formaz̧ione di una Regione Urbana, Roma, 2008.

L. De Propris, C. Chapain, P. Cooke, S. MacNeil, J. Mateos-Garcia, The Geography of Creativity, London, 2009.

A. L. PALAzZO (a cura), Campagne urbane: paesaggi in trasformazione nell'area romana, Roma, 2005.

E. PIRODDI, « Roma città regionale. Lazio regione urbana in via di sviluppo », Edilizia Popolare 284-285, 2010. 